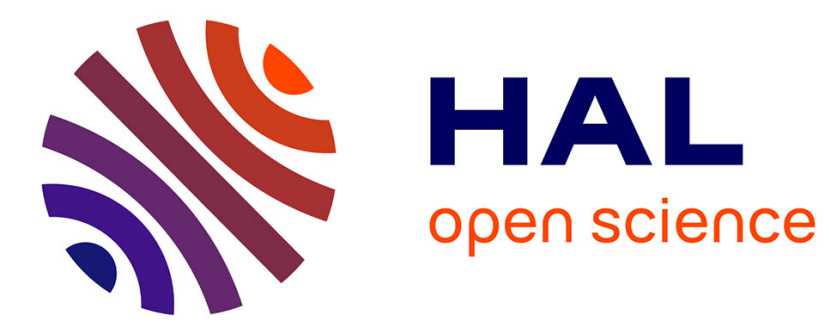

\title{
LOCALISATION SÉLECTIVE DE SOURCES ACOUSTIQUES
}

Pierre Bertrand

\section{To cite this version:}

Pierre Bertrand. LOCALISATION SÉLECTIVE DE SOURCES ACOUSTIQUES. Journal de Physique Colloques, 1990, 51 (C2), pp.C2-675-C2-678. 10.1051/jphyscol:19902157 . jpa-00230459

\section{HAL Id: jpa-00230459 https://hal.science/jpa-00230459}

Submitted on 1 Jan 1990

HAL is a multi-disciplinary open access archive for the deposit and dissemination of scientific research documents, whether they are published or not. The documents may come from teaching and research institutions in France or abroad, or from public or private research centers.
L'archive ouverte pluridisciplinaire HAL, est destinée au dépôt et à la diffusion de documents scientifiques de niveau recherche, publiés ou non, émanant des établissements d'enseignement et de recherche français ou étrangers, des laboratoires publics ou privés. 
COLLOQUE DE PHYSIQUE

Colloque C2, supplément au $\mathrm{n}^{\circ} 2$, Tome 51, Février 1990

ler Congrès Français d'Acoustique 1990

LOCALISATION SÉLECTIVE DE SOURCES ACOUSTIQUES

P. BERTRAND

Office National d'Etudes et de Recherches Aérospatiales, BP. 72 , F-92322 Châtilion Cedex, France

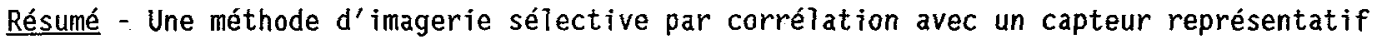
des sources est présentée. Appliquée d'abord à deux sources incohérentes en chambre sourde, elle a permis de les localiser l'une après l'autre, enfin, au cours d'une expérience en soufflerie, une source masquée par des bruiteurs a pu être mise en évidence.

Abstract - A selective array processing method using the correlation with a sensor representative of the noise source is presented. Experimental results show that it is likely to localize independentiy two incoherent sources and that it can identify an aerodynamic source completly hidden by strong hammers.

\section{1 - INTRODUCTION}

La caractérisation et la localisation de sources acoustiques d'origine aérodynamique en champ proche de $l^{\prime}$ antenne sont des problèmes qui se posent fréquemment lors de mesures en soufflerie. Si, ces dernières années, d'importants progrès ont été effectués dans la mâ̂trise des bruits de fond ou dans la forme des mâts supports, it n'en reste pas moins que, parfois, leur seule présence-dégrade la localisation, par des moyens classiques de focalisation $d$ 'antenne, de sources sonores de niveau faible. Une solution, propre au champ proche, consiste à utiliser des capteurs disposés sur le profil à mesurer, afin de sélectionner dans le champ ambiant 1 a source objet de l'étude.

Cet article présente une application d'une de ces méthodes $d^{\prime}$ imagerie sélective. Dans un premier temps, on montre que ce traitement peut se ramener à la focalisation classique des coefficients d'intercorrélation entre les capteurs de 1 'antenne et le capteur de référence. Puis cette démarche simplifiée est validée sur une expérience de base en chambre sourde.

Enfin, une application en soufflerie permet de mettre en évidence une source masquée par des sources parasites fortes.

\section{2 - IMAGERIE SELECTIVE}

\section{Principe général}

Les traitements de localisation sélective sont fondés sur la corrélation entre le signal d'antenne focalisée et le signal d'un capteur de référence. Cette corrélation étant maximale quand le point de focalisation coincide avec la source [1], on obtient bien un estimateur de position.

Cette procédure est longue et très couteuse en temps que calcul, puisqu'il faut balayer $l^{\prime}$ ensemble de la zone de focalisation pour chaque bloc temporel d'analyse. Au prix d'hypothèses simples, on peut cependant montrer qu'il est possible de réduire considérablement le temps de calcul sans pour autant dégrader les performances du traitement.

\section{Imagerie sélective simplifiée}

On suppose, d'une part, que 1'ensemble des signaux à traiter est stationnaire, en particulier que les sources sont fixes par rapport à $1^{\prime}$ antenne, d'autre part, que les 
retards de propagation entre le signal de référence et les signaux d'antenne sont bien inférieurs à la durée $T$ du bloc temporel $d^{\prime}$ analyse.

Le traitement simplifié consiste alors à calculer d'abord la corrélation entre les signaux de chaque capteur de l'antenne et le signal de référence, sur le nombre de moyennes voulu, et ensuite seulement d'effectuer, en une seule étape, la focalisation de ces corrélations. on montre alors $[2,3]$ que le biais relatif sur l'estimation des corrélations $\widehat{c}$ est de l'ordre de :

$$
\frac{E(\widehat{c})-c}{C} \approx-2 \frac{|D|}{T} \text { avec } \quad D \ll T
$$

où c est la corrélation à retard nul et $\mathrm{D}$ le retard de propagation d'un. capteur de l'antenne à celui de référence.

En pratique, $E(\widehat{c})$ est obtenu par moyennage temporel. Il suffit alors de prendre une taille de bloc suffisante, par exemple, telle que $2 \frac{1 D I}{T}<20 \%$, pour que les corrélations soient correctement estimées pour les besoins de l'imagerie.

\section{3 - RESULTATS EXPERIMENTAUX}

\section{Validation sur des sources électroacoustiques}

La figure 1 présente 1 'image classique de deux sources distantes de $2,75 \mathrm{~m}$ ' une antenne à 11 capteurs, alimentées en bruits blancs décorrélés filtrés dans la bande $[0,5 \mathrm{kHz}-5 \mathrm{kHz}]$. Les sources sont retrouvées à leur position à partir de $2 \mathrm{kHz}$; aux fréquences inférieures, la résolution de 1'antenne est insuffisante pour les séparer.

Sur les figures 2 et 3 sont portées respectivement les images en corrélation avec le signal de l'une puis de l'autre source. Celles-ci sont identifiées pour toutes les fréquences d'émission à leur position avec une cohérence comprises entre 30 et $50 \%$. La taille élémentaire du bloc d'analyse est de 512 points, soit $64 \mathrm{~ms}^{-a ̀ ~} 1 \mathrm{a}$ fréquence d'échantillonnage de $8 \mathrm{kHz}$. Le biais relatif théorique (1) est égal à $25 \%$, mais en pratique, le résultat global pour toute 1 'antenne apparaît comme meilleur.

\section{Mesures en soufflerie}

Les figures 4 et 5 montrent une application de cette méthode simplifiée à un profil situé dans un écoulement lors d'une campagne d'essais en soufflerie. L'image classique de la figure 4 montre que les sources les plus importantes sont localisées sur le mât support et sur le convergent de la soufflerie dont on ne voit sur l'image que le repliement spatial. La source attendue vers $5 \mathrm{~m}$ se trouve masquée et $n^{\prime}$ est donc pas localisée.

La figure 5 présente 1 'image sélective effectuée sur 2000 moyennes, avec un accéléromètre placé sur le bord de fuite du profil. Les sources parasites ont pratiquement disparues, seule apparaît, avec une corrélation très faible mais significative en terme de localisation, une source au droit du profil.

\section{4 - CONCLUSION}

Une technique simplifiée d'imagerie sélective a été présentée. Le gain en temps de calcul est très important puisque la focalisation s'effectue en une seule étape à partir des corrélations des signaux d'antenne avec un capteur de référence. Enfin, des résultats expérmentaux ont été présentés, qui ont validé la méthode et ont permis de localiser une source d'origine aérodynamique masquée par des bruiteurs de forte amplitude.

\section{REFERENCES BIBLIOGRAPHIQUES}

[1] Garcia P. et Gérard P., Revue d'Acoustique, 73 (1985) 270-293.

[2] Carter, C.G., IEEE, Trans. Acoust. Speech and Signal Process. ASSP-28 (1980), 97-99.

[3] Halvorsen, W.G. and Bendat, S.S., J. Sound and Vib. 1 (1975), 15-24. 


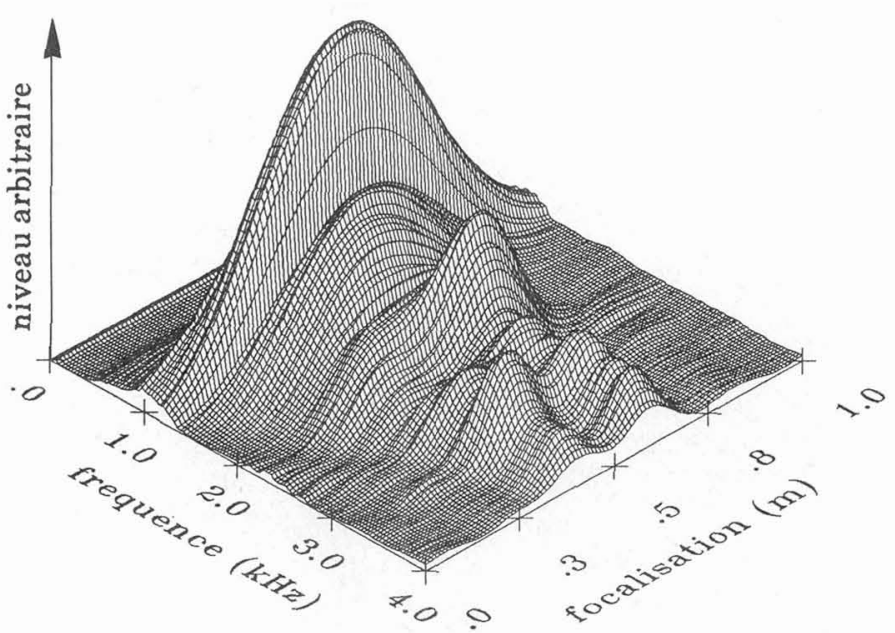

Fig. 1 - Image de 2 sources décorrélées.

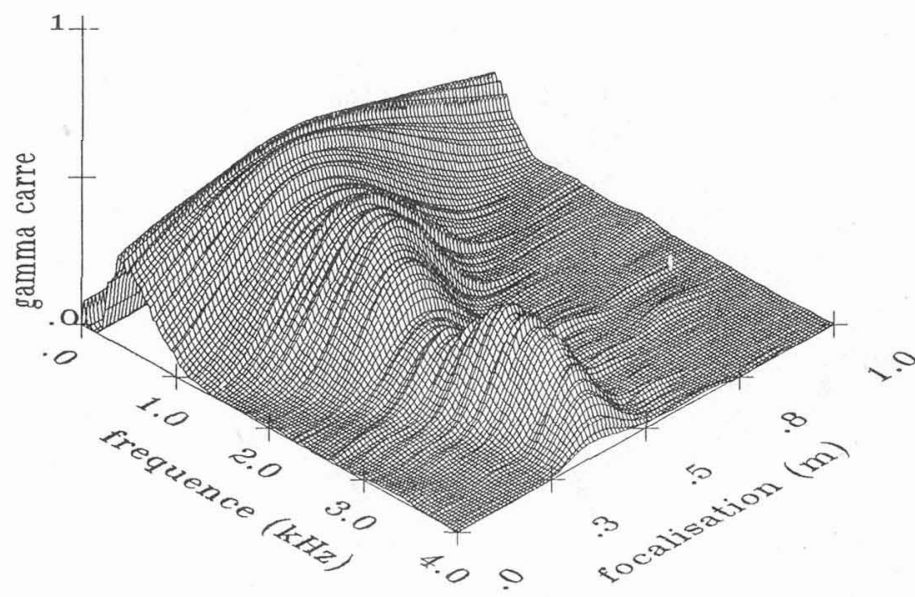

Fig. 2 - Image sélective, corrélation avec le signal 1.

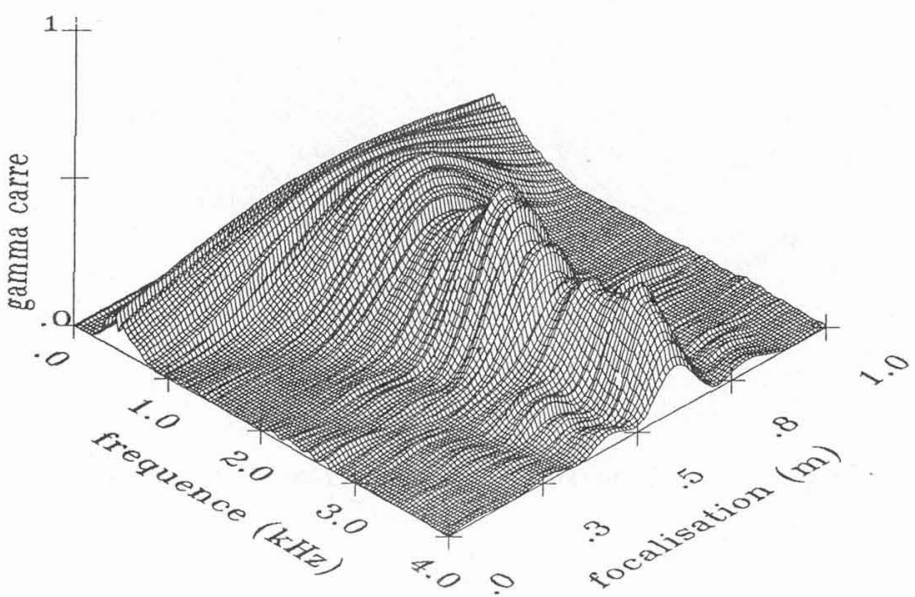

Fig. 3 - Image sélective, corrélation avec le signal 2. 


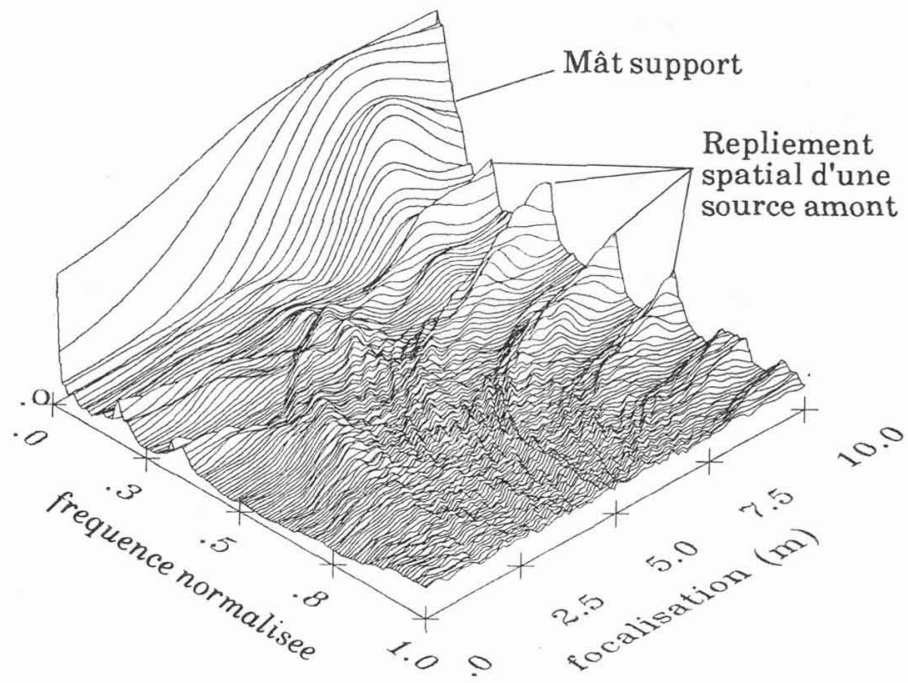

Fig. 4 - Image classique du profil.

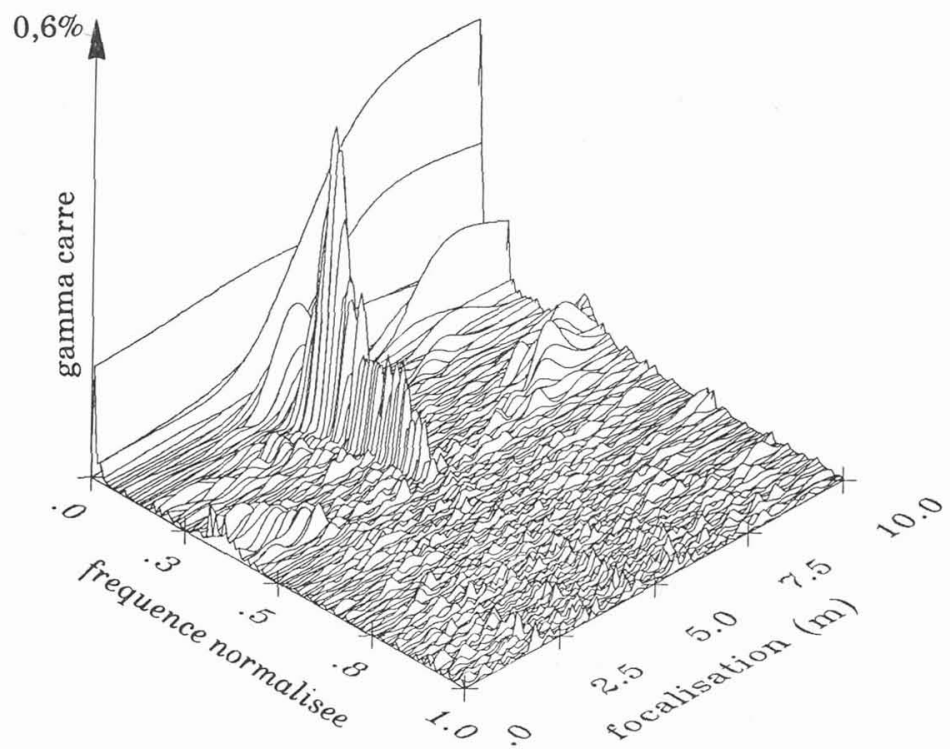

Fig. 5 - Image sélective avec signal de référence. 